\title{
THE EXTENSION AND THE LIFTING PROPERTIES OF BANACH SPACES
}

\author{
MORISUKE HASUMI ${ }^{1}$ AND G. L. SEEVER ${ }^{2,3}$
}

1. Statement of the theorem. The extension property and the lifting property of Banach spaces are dual concepts (cf. [7]). Therefore it is natural to raise the following questions:

(a) If a Banach space $E$ has the lifting property, then does the dual $E^{*}$ have the extension property?

(b) If $E$ has the extension property, then does $E^{*}$ have the lifting property?

In view of the well-known results on these two properties (see (I) and (II) of the next section) the answer to question (a) is clearly affirmative. The purpose of this note is to give an answer to question (b) in the following form:

Theorem. Suppose $E$ has the extension property. Then $E^{*}$ has the lifting property if and only if $E$ is finite dimensional.

2. Some preliminary results. Let $E$ be a Banach space. $E$ has the extension property if for any Banach space $F$, any closed linear subspace $S$ of $F$, and any bounded linear mapping $\phi$ of $S$ into $E$, there exists a linear extension $\Phi$ of $\phi$ to $F$ with values in $E$ and with the same norm. On the other hand, a Banach space $E$ has the lifting property if for any Banach space $F$ and any closed linear subspace $S$ of $F$, the natural mapping of $L(E, F)$ into $L(E, F / S)$ given by $\psi \rightarrow \pi \circ \psi$ (where $\pi$ denotes the natural mapping of $F$ onto $F / S$ ) is onto and of norm one. Concerning these properties, the following theorems are well known [2], [3], [4], [5], [6]:

(I) A real (or complex) Banach space $E$ has the extension property if and only if $E$ is isometrically isomorphic to the Banach space $C(K, R)$ (or $C(K, C)$ ) of all real (or complex) continuous functions with supremum norm on some Stonian space $K$.

(II) A real (or complex) Banach space $E$ has the lifting property if and only if $E$ is isometrically isomorphic to the Banach space $l^{1}(I, R)$ (or $l^{1}(I, C)$ ) of all real (or complex) summable functions with $l^{1}$-norm on some set $I$.

Received by the editors April 9, 1963 and, in revised form, May 16, 1963.

${ }^{1}$ On leave from Ibaraki University, Japan. Partly supported by the National Science Foundation, Grant NSF G-18974.

2 Partly supported by the Air Force Office of Scientific Research, Grant 62-140.

${ }^{3}$ The authors are indebted to the referee for informing them of the PelczyniskiSemadeni paper [8]. 
Next we mention some results on nonatomic measures on compact spaces. Pełczyński and Semadeni [8] showed, among others, that a compact Hausdorff space $X$ has a nonzero, nonatomic, regular, Borel measure if and only if $X$ contains a nonvoid perfect subset ( $=$ a closed subset which is, as a subspace, without isolated points). A space is Stonian if it is a compact Hausdorff space in which any two disjoint open sets have disjoint closures. We then have

Lemma. If $X$ is an infinite Stonian space, then the set $X_{0}$ of nonisolated points of $X$ is perfect.

Proof. Suppose that $X_{0}$ is not perfect. Then there exists a point $x \in X_{0}$ and a closed neighborhood $U$ of $x$ such that $U \cap X_{0}=\{x\}$. Since $x$ is not isolated in $X, U$ is an infinite set. Moreover every point of $U \sim\{x\}$ is an isolated point. It follows that $U$ is the one-point compactification of the discrete space $U \sim\{x\}$. But, since $X$ is Stonian, $U$ is also the Stone-Čech compactification of $U \sim\{x\}$, and this is impossible. Hence $X_{0}$ must be perfect.

Thus by Pełczyński-Semadeni's theorem we have the following:

(III) Every infinite Stonian space has a nonzero, nonatomic, regular Borel measure.

3. Proof of the theorem. By (I), $E$ is isometrically isomorphic with $C(K)$ for some Stonian space $K$, and therefore $E^{*}$ is isometrically isomorphic with $C(K)^{*}$ (= the space of real (or complex) regular, Borel measures on $K$ ). If we suppose that $E^{*}$ has the lifting property, then by (II) $E^{*}$ is isometrically isomorphic with $l^{1}(I)$ for some set $I$. The unit ball of $l^{1}(I)$ is the norm-closed convex hull of its extreme points. Therefore $C(K)^{*}$ enjoys the same property. The extreme points of the unit ball of $C(K)^{*}$ are of the form $\alpha e_{x}$, where $|\alpha|=1$, and $e_{x}$ is the evaluation at $x \in K$ (cf. [1]). The norm-closed convex hull of such points is precisely the set of purely atomic measures of norm not exceeding one, i.e., $K$ has no nonatomic measures except zero. Therefore $K$ is finite by (III). The other half of the theorem is trivial.

\section{REFERENCES}

1. R. F. Arens and J. L. Kelley, Characterization of the space of continuous functions over a compact Hausdorff space, Trans. Amer. Math. Soc. 62 (1947), 499-508.

2. D. B. Goodner, Projections in normed linear spaces, Trans. Amer. Math. Soc. 69 (1950), 89-108.

3. A. Grothendieck, Une caractérisation vectorielle-métrique des escapes $L^{1}$, Canad. J. Math. 7 (1955), 552-561.

4. M. Hasumi, The extension property of complex Banach spaces, Tôhoku Math. J. (2) 10 (1958), 135-142. 
5. J. L. Kelley, Banach spaces with the extension property, Trans. Amer. Math. Soc. 72 (1952), 323-326.

6. L. Nachbin, $A$ theorem of the Hahn-Banach type for linear transformations, Trans. Amer. Math. Soc. 68 (1950), 28-46.

7. - Some problems in extending and lifting continuous linear transformations, Proc. Internat. Sympos. Linear Spaces (Jerusalem, 1960), pp. 340-351, Jerusalem Academic Press, Jerusalem, 1961.

8. A. Pełczyński and Z. Semadeni, Spaces of continuous functions. III, Studia Math. 8 (1959), 211-222.

UNiversity of CALIForNia, BERKELEy

\section{GEODESICS AND LEBESGUE AREA}

\section{EDWARD SILVERMAN ${ }^{1}$}

A method of stretching a Fréchet surface by various amounts, without increasing its Lebesgue area, was introduced in [S2]. The stretched surfaces derived from a given continuous surface are all continuous and, in general, there is no largest such surface. In this paper we admit discontinuous surfaces and this enables us to stretch a surface to its maximum extension. If the maximum extension is continuous, then its (Lebesgue) area is the same as the area of the surface being stretched. In general, if the area of the maximum extension is defined to be the supremum of all continuous surfaces which are not bigger, in the sense of Kolmogoroff's Principle applied to surfaces, then this area of the maximum extension of a given continuous surface agrees with the Lebesgue area of the surface. Furthermore, if two continuous surfaces are so related that the pseudo-geodetic distances between corresponding pairs of points are equal, then the maximum stretched surfaces derived from these two are isometric, which implies that the two original surfaces have the same Lebesgue area. (The pseudo-geodetic distance agrees with geodetic distance for light maps.) An example of R. D. Anderson, modified by R. F. Williams, gives a monotone map of a square onto another square with the property that the geodetic distance between every distinct pair of points is infinite. Thus, it is neither desirable nor possible for the preceding result to hold for geodetic as well as for pseudo-geodetic distance.

Presented to the Society, August 30, 1962; received by the editors April 25, 1963.

1 This research was supported in part by National Science Foundation Grant No. GP634. 